\title{
COMUNIDADES DE MICROMAMÍFEROS DE BOSQUE SERRANO Y PASTIZAL DE ALTURA EN LA SIERRA CHICA, CORDOBA, ARGENTINA
}

\section{SMALL MAMMAL COMMUNITIES OF MOUNTAIN FOREST AND GRASSLAND IN SIERRA CHICA, CORDOBA, ARGENTINA}

\author{
Mariana Altrichter ${ }^{1}$, Maura Kufner ${ }^{2}$, Liliana Giraudo ${ }^{3}$, Gregorio Gavier ${ }^{3}$, Daniela Tamburini ${ }^{2}$, Mariano Sironi ${ }^{3}$ y \\ Liliana Arguello ${ }^{2}$
}

\section{Resumen}

Las comunidades de micromamíferos en las Sierras Chicas, Córdoba, Argentina se estudiaron en cuanto a su composición, abundancia relativa, diversidad y relación con la estructura del hábitat según la cobertura vegetal, en el pastizal de altura y el bosque serrano de la Reserva La Quebrada. Se muestreó mediante cuadrículas de 30 trampas, durante tres noches consecutivas por estación, desde primavera de 1991 a invierno de 1993. Se capturaron 216 ejemplares correspondientes a siete especies de la familia Muridae y dos de la familia Didelphidae. La reserva es zoogeográficamente representativa de la mastofauna serrana y tiene composición particular respecto de otras localidades provinciales. La composición específica fue diferente entre ambos ambientes. El pastizal de altura fue el hábitat más favorable para la comunidad de micromamíferos; de seis especies $A$. azarae, $N$. benefactus y $M$. dimidiata presentaron mayor contribución. En el bosque $P$. xanthopygus concentró el $85 \%$ de las capturas. La abundancia relativa y la diversidad específica fueron más altas en el pastizal. Múridos aumentaron en otoñoinvierno y Didélfidos en verano-otoño. En el uso del hábitat se destacó el estrato de cobertura gramíneo-herbácea del pastizal entre 0.11 y 0.40 metros, como predictor de la abundancia de micromamíferos.

Palabras clave: Comunidades, Didelphidae, Muridae, Bosque serrano, Pastizal de altura, Sierras Chicas, Córdoba, Argentina.

\begin{abstract}
Composition, relative abundance, species diversity and relation with habitat structure of small mammal communities of the Sierras Chicas were studied in the grassland and forest vegetation layers of La Quebrada Reserve, Córdoba, Argentina. Traps were set in two 30 spot grids for three consecutive nights, seasonally from spring 1991 to winter 1993. Seven species of Muridae were captured: Akodon azarae, A. dolores, Calomys musculinus, C. venustus, Necromys benefactus, Phyllotys xanthopygus and Oxymycterus rufus, and two species of Didelphidae: Monodelphis dimidiata and Thylamys elegans. The reserve is zoogeographically representative of the Sierras' mammalian fauna, and it has a particular composition in respect of other provincial localities. Six species were caught in the grassland, with major contribution of Akodon azarae, Necromys benefactus. y Monodelphis dimidiata. P. xanthopygus was the most abundant species in the forest. Small mammal communities composition was different in both habitats (Czekanowki I. $=0.009$ ). Relative abundance and specific diversity were higher in grassland. Murids augmented in autumnwinter and Didelphids in summer-autumn. Habitat use related to vegetation cover analysis revealed forbs and grasses' (between 0.11 and $0.40 \mathrm{~m} \mathrm{high}$ ) as the most important vegetation layer to predict small mammals abundance $\left(\mathrm{R}^{2}=0.74 ; \mathrm{P}<0.04\right)$.
\end{abstract}

Key words: communities, Didelphidae, Muridae, mountain forest Bosque grassland, Sierras Chicas, Córdoba, Argentina.

\section{Introducción}

La mayoría de los estudios que se han hecho en Argentina sobre pequeños mamíferos silvestres están orientados hacia agroecosistemas (Kravetz et al., 1975; Kravetz, 1978; Polop et al., 1982) y son escasos los estudios que relacionan la distribución y abundancia de éstos con factores físicos y ecológicos en el medio natural (de Villafañe, 1970; Pearson 1983; Polop et al., 1985; Polop, 1989, 1991;
Bonaventura et al., 1995). En las áreas protegidas provinciales son pocos y relativamente recientes los estudios que tratan a la fauna como componente natural del sistema y analizan su biodiversidad y representatividad zoogeográfica (Kufner et al., 1998).

La estructura del hábitat influye en la organización y distribución de las comunidades de micromamíferos (Birney et al., 1976; Stenseth, 1980). En ambientes de montañas de otras latitudes se ha constatado variación 
de la diversidad específica con la altitud (Linares, 1995; Patton et al., 1990). En algunas zonas de las Sierras Chicas y Grandes de Córdoba, donde el bosque ha quedado relictual en las quebradas, Polop $(1989,1991)$ y Polop et al. (1985) encuentran similar riqueza de muroideos entre los pisos de vegetación. De éstos, particularmente el bosque y el pastizal proporcionan una variedad de hábitats y mantienen una importante diversidad faunística (Kufner et al., 1998).

El objetivo de este estudio fue analizar la composición y diversidad de las comunidades de pequeños mamíferos de la Sierra Chica, su variación en tiempo y espacio y su relación con la cobertura vegetal, en el bosque serrano y el pastizal de altura, en la Reserva La Quebrada, Córdoba, Argentina.

\section{Materiales y métodos \\ Área de estudio}

La Reserva La Quebrada protege 4200 ha de la cuenca hídrica del Río Ceballos en las Sierras Chicas del Departamento Colón, Córdoba, Argentina, entre los 800 y 1350 msnm (Luti et al., 1979). El relieve está constituido por sierras antiguas, bajas, con cumbres redondeadas y quebradas y valles modelados por arroyos. El clima es subhúmedo mesotermal según Thornwaite (Papadakis, 1956). El régimen de precipitaciones es continental monzónico, con mayor concentración de lluvias entre Octubre y Marzo. Su valor medio anual es de $750 \mathrm{~mm}$ y el de la temperatura de $13^{\circ} \mathrm{C}$ (Estrabou, 1986). Las variaciones de altitud y relieve determinan microclimas que permiten el desarrollo de unidades fisonómicas o pisos de vegetación definidos: Bosque serrano (750 a $850 \mathrm{~m})$, con los dominantes Lithraea ternifolia, Fagara coco, Acacia caven, Stipa pseudoichu; Arbustal (1000 a $1100 \mathrm{~m})$ con Celtis pallida, Heterothalamus alienus, Colletia spinosisima y Pastizal de Altura (desde los 1000 m) con vegetación herbácea de gramíneas predominante como Festuca hieronymi, Stipa trichotoma, Paspalum dilatatum, entre otras. El estudio se llevó a cabo en ambientes homogéneos de bosque serrano y pastizal de altura, cuyos respectivos aportes faunísticos serían principalmente chaqueños y andino-patagónicos (Ringuelet, 1961; Bucher \& Abalos, 1979).

\section{Metodología}

Se seleccionaron áreas homogéneas, no perturbadas y representativas de la vegetación característica donde se ubicaron al azar dos cuadrículas de trampeo: una en bosque y otra en pastizal. Cada una constó de 30 trampas tipo Sherman, distanciadas entre sí $10 \mathrm{~m}$, que se activaron estacionalmente durante tres noches consecutivas, desde primavera de 1991 hasta invierno de 1993. La identificación taxonómica fue realizada en base a reconocimiento de cráneos, pieles y análisis electroforéticos. Se siguió la nomenclatura propuesta por Galliari et al. (1996). Los especímenes fueron depositados en la colección del Museo de Zoología, FCEF y Naturales, UNC. La composición de las comunidades se determinó a partir del registro de todas las especies. El número de individuos capturados se tomó como índice de abundancia relativa, ya que el esfuerzo de muestreo se mantuvo constante. La similitud de la composición de las comunidades de micromamíferos se comparó mediante el índice cuantitativo de Czekanowski: $C Z j k=2 \min (X i j, X i k) /(X i j+X i k)$ (Clifford \& Stephenson, 1975). La diversidad específica fue estimada a través del índice de Shannon (Magurran, 1991). La utilización del hábitat se investigó tomando como índice la abundancia relativa de cada especie en pastizal y bosque (M'Closkey \& Fieldwick, 1975), relacionándolo con la estructura del hábitat. Para ello se estimó la cobertura vegetal comprendida entre el suelo y un metro de altura según el método de Canfield (Hays et al., 1981); los estratos de vegetación considerados fueron los siguientes: $\mathrm{E} 1=0$ a $0.10 \mathrm{~m}$ (mantillo y suelo desnudo), E2 $=0.11$ a 0.40 $\mathrm{m}$ (vegetación herbácea) y E3 $=0.41$ a $1.00 \mathrm{~m}$ (vegetación arbustiva). Mediante un análisis de regresión múltiple seleccionada se relacionó la variable dependiente "abundancia de micromamíferos" con la independiente "cobertura de estratos vegetales", para seleccionar las variables predictoras más significativas en la ecuación (Zar, 1984). Se trabajó con todos los datos ordenados estacional y correlativamente, en un único análisis.

\section{Resultados y discusión \\ Composición específica}

Se capturó un total de 216 micromamíferos durante 1440 noches trampa, identificándose nueve especies: siete Múridos: Akodon azarae (Fischer, 1829), A.dolores Thomas, 1916, Calomys musculinus (Thomas, 1913) C. venustus (Thomas, 1894), Necromys benefactus (Thomas, 1916), Phyllotys xanthopygus (Waterhouse, 1837), Oxymycterus rufus (Fischer, 1814) y dos Didélfidos: Monodelphis dimidiata (Wagner, 1847) Thylamys elegans (Waterhouse, 1839)

El número total de individuos capturados fue mayor en pastizal (84\%) que en bosque (16\%) (Tabla 1). En el pastizal $A$. azarae fue la especie mejor representada $(38 \%)$, seguida en importancia por $N$. benefactus (28\%). En general, la composición de la comunidad del pastizal constó de dos o más especies y relativa variación de sus proporciones estacionales. El bosque serrano tuvo una representación específica diferente y con mayor proporción relativa de $P$. xanthopygus $(85 \%)$, alcanzando las otras especies sumadas sólo $15 \%$. 
Tabla 1. Composición específica y número de pequeños mamíferos en hábitats de pastizal $\mathrm{y}$ bosque.

Small mammals community specific composition and representativeness in grassland and mountain forest.

\begin{tabular}{|c|c|c|c|}
\hline FAMILIA & ESPECIE & 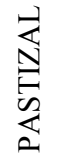 & 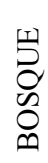 \\
\hline \multirow[t]{7}{*}{ Muridae } & Akodon azarae & 69 & 0 \\
\hline & A. dolores & 0 & 2 \\
\hline & Calomys musculinus & 1 & 0 \\
\hline & C. venustus & 0 & 2 \\
\hline & $N$. benefactus & 51 & 0 \\
\hline & Oxymycterus rufus & 29 & 0 \\
\hline & Phyllotis xanthopygus & 1 & 28 \\
\hline \multirow[t]{2}{*}{ Didelphidae } & Monodelphis dimidiata & 32 & 0 \\
\hline & Thylamys elegans & 0 & 1 \\
\hline TOTAL & & 183 & 33 \\
\hline
\end{tabular}

La fauna de micromamíferos de La Quebrada en las Sierras Chicas, además de justificar la representatividad zoogeográfica chaqueña y andinopatagónica atribuida a las sierras (Bucher \& Abalos, 1979), presentó una composición particular con respecto a otras localidades de Córdoba. La especie andina $P$. xanthopygus y la subtropical $O$. rufus, han sido citadas en pocas oportunidades en la provincia y en ámbitos diferentes a nuestra área de estudio (Crespo et al., 1970; Olrog \& Lucero, 1981; Kravetz \& Polop, 1983; Polop et al., 1982, 1985; Polop, 1989). Si bien el límite altitudinal señalado en las Sierras Grandes para O. rufus serían los $800 \mathrm{msnm}$ (Polop, 1991), en nuestro estudio este roedor se encontró en números elevados a más de 1000 msnm. La dominancia de $A$. azarae en pastizal de altura es otro rasgo distintivo, ya que el pastizal pampeano se ha descripto como su hábitat característico (Crespo, 1966; de Villafañe et al., 1977) y en otras localidades de las sierras esta especie ha sido hallada en menor número (Polop et al., 1985). Tampoco N. benefactus ha sido citado frecuentemente en ambientes serranos (Polop et al., 1985; Polop, 1989, 1991; Priotto et al., 1996). La presencia en proporción elevada del marsupial $M$. dimidiata, de origen parano-brasílico, también aportó un carácter diferencial; éste había sido citado por Reig (1964) y Bush \& Kravetz (1991) como elemento frecuente en el noreste y suroeste de la provincia de Buenos Aires. Por su parte, la escasa representación del género Calomys podría indicar buen estado del área de estudio dado que sería abundante en hábitats perturbados (de Villafañe et al., 1977).
Abundancia relativa, similitud $y$ diversidad específica de las comunidades de pastizal y bosque.

Se capturaron 5.5 veces más individuos en el pastizal que en el bosque (Tabla 1); el número total de individuos capturados resultó mayor en pastizal ( $t=$ $3.9 \mathrm{P}<0.001$, g.l. $=7$ ) y no difirió significativamente entre los dos años de estudio $(t=1.22 \mathrm{P}=0.24$, g.l.= 6). En pastizal la abundancia relativa de múridos presentó la variación estacional característica del grupo (Tabla 2) con aumento invernal -asignado por Crespo et al. (1970) y Polop et al. (1985) al reclutamiento de juveniles en el período reproductivo aproximadamente entre Setiembre y Junio y disminución estival por comportamiento expansivo de animales en estado reproductivo (Krebs \& Boonstra, 1978). La baja abundancia de múridos en el bosque no permitió este análisis. Los marsupiales, representados casi exclusivamente por $M$. dimidiata, mostraron un aumento en verano-otoño, éste no coincidió con el pico primaveral de la especie en la provincia de Buenos Aires (Reig, 1964). El retraso comparativo podría relacionarse con la semiaridez de la región serrana cordobesa.

Tabla 2. Abundancia relativa estacional de Múridos y Didélfidos en pastizal de altura y en bosque serrano.

Seasonal abundance of Murids and Didelfids in grassland and muntain forest of Sierra Chica.

\begin{tabular}{|c|c|c|c|c|}
\hline PASTIZAL & 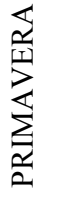 & $\frac{o}{\not}$ & \begin{tabular}{l}
0 \\
\hdashline \\
0 \\
0 \\
0
\end{tabular} & 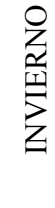 \\
\hline Múridos & 27 & 21 & 30 & 73 \\
\hline Didélfidos & 2 & 12 & 13 & 5 \\
\hline \multicolumn{5}{|l|}{ BOSQUE } \\
\hline Múridos & 3 & 5 & 12 & 12 \\
\hline Didélfidos & 0 & 0 & 1 & 0 \\
\hline
\end{tabular}

Tabla 3. Cobertura vegetal media de tres estratos: E1, E2 y E3 de pastizal y bosque, estival e invernal, en la Reserva La Quebrada.

Cover of three vegetation layers (E1, E2 y E3) in forest and grassland during summer and winter periods, in La Quebrada reserve.

\begin{tabular}{|c|c|c|c|c|c|}
\hline \multirow{3}{*}{ Estrato } & \multirow{3}{*}{$\begin{array}{c}\text { Altura } \\
(\mathrm{m})\end{array}$} & \multicolumn{4}{|c|}{ Cobertura vegetal media (\%) } \\
\hline & & \multicolumn{2}{|c|}{ Verano } & \multicolumn{2}{|c|}{ Invierno } \\
\hline & & Pastizal & Bosque & Pastizal & Bosque \\
\hline E1 & $0.00-0.10$ & 2.59 & 4.05 & 9.88 & 6.61 \\
\hline E2 & $0.11-0.40$ & 46.68 & 21.09 & 82.52 & 6.70 \\
\hline E3 & $0.41-1.00$ & 50.72 & 24.35 & 7.62 & 16.60 \\
\hline
\end{tabular}


Las comunidades de micromamíferos de pastizal y bosque presentaron composiciones específicas disímiles $(\mathrm{CZjk}=0.009)$. La diversidad total de la comunidad de micromamíferos resultó significativamente más elevada en el pastizal $(\mathrm{H}=1.37762)$ que en el bosque $(\mathrm{H}=0.58517)(\mathrm{t}=4.3$, g.l $=36,43 ; \mathrm{P}<0.01)$. Ello indicaría que el pastizal presenta recursos de interés para mayor número de especies. La limitación de recursos haría del bosque un hábitat favorable para pocos micromamíferos; Bonaventura et al. (1991) asumen que baja cobertura herbácea y alto porcentaje de suelo descubierto relativos (Tabla 3), provocarían esa condición.

\section{Uso del hábitat}

El pastizal fue el hábitat más utilizado, tanto en referencia al número de individuos como al de especies. Entre los micromamíferos detectados allí predominaron las especies caminadoras del suelo sobre las arborícolas, lo que indicaría una relación entre características morfológicas y la utilización del microhábitat (Rosenzweig \& Winakur, 1969; Murúa et al., 1987). Las especies que resultaron abundantes en el pastizal, $A$. azarae, $N$. benefactus y $O$. rufus son terrícolas, de hábitos cavícolas y ocupan hábitats con predominio de gramíneas y alta cobertura herbácea (Cabrera \& Yepes, 1960; de Villafañe et al., 1977; Bonaventura et al., 1991). El marsupial M. dimidiata camina en el suelo y es asociado comúnmente con hábitats de tipo pastizal (Cabrera \& Yepes, 1960; Reig 1964). Encontrada como dominante en el bosque, $P$. xanthopygus es arborícola, anida bajo piedras y tiene preferencia por lugares con roca expuesta (Cabrera \& Yepes, 1960) como en La Quebrada.

En cuanto a la relación entre la estructura y la utilización del hábitat, el estrato E2 $(0.11$ a $0.40 \mathrm{~m})$ fue seleccionado en la regresión como predictor de la abundancia de micromamíferos; la ecuación que relacionó ambas variables fue la siguiente: $\mathrm{y}=-1.03+$ 0.71 E2 $\left(\mathrm{R}^{2}=0.74 ; \mathrm{P}<0.05 ;\right.$ g.l.= 1, 7). El valor de dicho estrato reside en que tiene un alto porcentaje de cobertura, especialmente en invierno (Tabla 3). Distintos autores han referido la importancia de la cobertura vegetal gramíneo-herbácea en micromamíferos de distintas latitudes (Rosenzweig \& Winakur, 1969; Ostfeld, 1985; Bonaventura \& Kravetz, 1989). La cobertura vegetal es aparentemente uno de los recursos del hábitat más importantes para los micromamíferos, influyendo en la disponibilidad de alimento, sitios de nidificación, refugio y protección contra la predación (Getz, 1965; Birney et al., 1976; Belk et al, 1988).

\section{Conclusiones}

La Reserva La Quebrada es zoogeográficamente representativa de la mastofauna serrana. Por primera vez se citan A. azarae, O. rufus, $N$. benefactus y $M$. dimidiata, como especies constituyentes de la comunidad del pastizal de altura de las Sierras Chicas. El pastizal de altura resulta ser el hábitat más favorable para la comunidad de micromamíferos, múridos y marsupiales, en términos de composición, abundancia relativa y diversidad específica. Se destaca la importancia del estrato de cobertura gramíneoherbáceo del pastizal como predictor de la abundancia de micromamíferos de una variedad de especies en relación con recursos del hábitat.

\section{Agradecimientos:}

Al Ing. Marietti de la Dirección de Hidráulica por facilitarnos la vivienda del Dique; a la Dirección de Areas Naturales por apoyar nuestro trabajo; a Marcos Torres, J. Polop, N. von Müller y N. Gardenal por la identificación de especies; a N. Cech, A. Carranza, P. Villagra, R. Giadrosi y Florencia di Tada por colaborar en el muestreo; a I. Jimenez por la revisión del manuscrito.

\section{Literatura citada}

Belk M., Smith D. \& Lawson J. 1988. Use and partitioning of montane habitat by small mammals. Journal of Mammalogy. 69: 688-695.

Birney E., Grant W. \& Bird D. 1976. Importance of vegetative cover to cycles of Microtus populations. Ecology. 57: 1043-1051.

Bonaventura S. \& Kravetz F. 1989. Relación roedor vegetación: Importancia de la disponibilidad de cobertura verde para Akodon azarae durante el invierno. Physis (Buenos Aires), Secc. C. 47: 1-5.

Bonaventura S., Piantanida M., Gurinin L. \& Sanchez López M. 1991. Habitat selection in population of cricetine rodents in the Delta region (Argentina). Mammalia. 53: 339-354.

Bonaventura S., Balabusik A.M., Duco A.M., Ferrero F., Marcelino F., Miranda A.M \& Sabattini M.C. 1995. Preferencia de hábitat de una comunidad de pequeños roedores en el parque Nacional Lihuel Calel. La Pampa, Argentina. p. 24. Resúmenes del III Congreso Latinoamericano de Ecología, Mérida, Venezuela.

Bucher E. \& Abalos J. 1979. Fauna. Páginas 368434 en J. Vázquez, R. Miatello y E. Roqué, eds. Geografía Física de la Provincia de Córdoba. Editorial Boldt, Buenos Aires.

Bush M. \& Kravetz F. 1991. Diet composition of Monodelphis dimidiata (Marsupialia, Didelphidae). Mammalia. 55: 619-621.

Cabrera A. \& Yepes J. 1960. Mamíferos Sudamericanos. Tomo II. Ediar, Buenos Aires.

Clifford H. \& Stephenson W. 1975. An introduction to numerical classification. Academic Press. London.

Crespo J. 1966. Ecología de una comunidad de roedores silvestres en el partido de Rojas, Provincia de Buenos Aires. Rev Mus. Cien. Nat. 
"Bernardino Rivadavia" Ecología. 1: 79-134.

Crespo J., Sabatini M., Piantanida M. \& de Villafañe G. 1970. Estudios ecológicos sobre roedores silvestres. Observaciones sobre densidad, reproducción y estructura de comunidades de roedores silvestres en el sur de la Provincia de Córdoba. Ministerio de Bienestar Social, Secretaría de estado y Salud Pública, Buenos Aires, Argentina.

de Villafañe G. 1970. Ecología de roedores silvestres (Cricetidae) en el sur de la provincia de Córdoba, completada con observaciones sobre reproducción y desarrollo obtenidas por cría experimental. Tesis doctoral inédita. Universidad Nacional de Córdoba, Argentina.

de Villafañe G., Kravetz F., Donadio O., Percich R., Knechen L., Torres M. \& Fernandez N. 1977. Dinámica de las comunidades de roedores en agroecosistemas pampásicos. Medicina, Buenos Aires. 37: 128-138.

Estrabou C. 1986. Rasgos estructurales de la vegetación en relación a las características físicas del ambiente y la acción humana. Páginas 116-131 en MAB 6, Efecto de las actividades humanas sobre los ecosistemas montañosos y de tundra. ROSTLAC. Montevideo, Uruguay.

Galliari C.A., Pardiñas U.F. \& Goin F.J. 1996. Lista comentada de los mamíferos argentinos. Mastozoología Neotropical. 3: 39-61.

Getz L. 1965. Humidities in voles runways. Ecology. 46: 548-550.

Hays R., Summers C. \& William S. 1981. Estimating wildlife habitat variables. Fish \& Wildlife Service. FWS/OBS-81/47.

Kravetz F.O., de Villafañe G., Torres M.P. \& Piantanida M.J. 1975. Poblaciones de roedores en un campo de trigo. Physis. 34: 187-197.

Kravetz F.O. 1978. Ecología de las comunidades de roedores involucrados en la Fiebre Hemorrágica Argentina. Tesis Doctoral. Inédita. Univ. Nac. de Buenos Aires, Argentina.

Kravetz F. \& Polop J. 1983. Comunidades de roedores en agroecosistemas del departamento Río Cuarto, Córdoba. Ecosur. 10:1-18.

Krebs C.J. \& Boonstra R. 1978. Demography of the spring decline in populations of the vole Microtus townsendii. Animal Ecology. 47: 407-418.

Kufner M., Giraudo L., Gavier G., Tamburini D., Altrichter M. \& Sironi M. 1998. Fauna de tetrápodos y sus biótopos en la Reserva La Quebrada, Río Ceballos, Córdoba. Acta Zoológica Lilloana. 44(1): 177-184.

Linares O.J. 1995. Gradiente altitudinal y zonal de los mamíferos de los bosques lluviosos de la Guayana y Amazonía Venezolana: Causas Evolutivas y Ecológicas. p. 24. Resúmenes III Congreso Latinoamericano de Ecología. Facultad de
Ciencias Universidad de los Andes-Mérida, Venezuela.

Luti R., Solis M., Galera F., de Ferreyra N.M., Berzal M., Nores M., Herrera M. \& Barrera J. 1979. Vegetación. Páginas 297-367 en J. Vázquez, R. Miatello y E. Roqué, eds. Geografía Física de la Provincia de Córdoba. Editorial Boldt, Buenos Aires.

Magurran A. 1991. Ecological Diversity and its Measurement. Chapman and Hall, London.

M'Closkey R. \& Fieldwick B. 1975. Ecological separation of sympatric rodents Peromyscus and Microtus. Journal of Mammalogy. 56: 119-129.

Murua R., Meserve L., Gonzales L. \& Jofre C. 1987. The small mammal community of a Chilean temperate rain forest: lack of evidence of competition between dominant species. Journal of Mammalogy. 68: 729-738.

Olrog C. \& Lucero M. 1981. Guía de los mamíferos argentinos. Fundación M. Lillo, Argentina.

Ostfeld R. 1985. Limiting resources and territoriality in microtine rodents. American Naturalist. 126: 115.

Papadakis J. 1956. Informe ecológico sobre la provincia de Córdoba. Instituto Suelos y Geotécnica. Publicación No 48. Buenos Aires.

Patton J.L., Myers P. \& Smith M.F. 1990. Vicariant versus gradient models of diversification: The small mammal fauna of easter Andean slopes of Perú. Páginas 355-371 en G., Peters y R. Hutterer, eds. Vertebrates in the tropics. Museum Alexander Koenig, Bonn.

Pearson O.P. 1983. Characteristiccs of a mammalian fauna from forests in Patagonia, saouthern Argentina. Journal of Mammalogy. 64: 476-492.

Polop J., Gardenal \& Sabattini M. 1982. Comunidades de roedores de cultivos de sorgo en la Provincia de Córdoba y su posible vinculación con la Fiebre hemorrágica Argentina. Ecosur. 9: 107-116.

Polop J., Martinez R. \& Torres M. 1985. Distribución y abundancia de poblaciones de pequeños roedores en la zona de Embalse de Río Tercero, Córdoba. Historia Natural. 5: 33-44.

Polop J. 1989. Distribution and ecological observations of wild rodents in Pampa de Achala, Córdoba, Argentina. Studies on Neotropial Fauna and Environment. 24:53-59. 1991. Distribución de Cricétidos en las sierras de Achala (Provincia de Córdoba, República Argentina). Rev. UNCR. 11: 115-121.

Priotto J.W., Morando M. \& Avila L. 1996. Nuevas citas de roedores de los pastizales de altura de la sierra de Comechingones, Córdoba, Argentina. Facena 12:135-137.

Reig O. 1964. Roedores y marsupiales del partido de General Pueyrredón y regiones adyacentes (Provincia de Buenos Aires, Argentina). 
Publicaciones del Museo Municipal de Ciencias Naturales de Mar del Plata. 1: 203-224.

Ringuelet R. 1961. Rasgos fundamentales de la zoogeografía de la Argentina. Physis (Buenos Aires). 22: 151-170.

Rosenzweig M. \& Winakur J. 1969. Population ecology of desert rodent communities: habitats and environmental complexity. Ecology. 50: 558-752.
Stenseth N. 1980. Spatial heterogeneity and population stability: some evolutionary consequences. Oikos. 35: 165-184.

Zar J. 1984. Biostatistical analysis. Prentice \& Hall. New Jersey.

\footnotetext{
${ }^{1}$ Department of Zoology. University of Wisconsin, Madison. 1202 W. Johnson St. Madison, WI 53706.

${ }^{2}$ Centro de Ecología y Recursos Naturales Renovables. Universidad Nacional de Córdoba. Av. Velez Sársfiel 299. 5000 Córdoba, Argentina. * CONICET.

${ }^{3}$ Cátedra de Diversidad Animal II. Facultad de Ciencias Exactas, Físicas y Naturales. Universidad Nacional de Córdoba. Av. Vélez Sársfield 299. 5000 Córdoba, Argentina.
}

Correspondencia a: Maura B. Kufner, Echeverría (S) 343. 5003 Alto Alberdi, Córdoba. Correo electrónico: bkufner@com.uncor.edu 